\title{
Application of the One-Stop Cardiac CT Scan on 256-Row Wide Detector Revolution CT in Myocardial Ischemia of Aortic Valve Degeneration Diagnosis
}

\author{
Xuefang $\mathrm{Lu}^{1,{ }^{*}}$, Wei Gong ${ }^{1}$ and Yunfei Zha ${ }^{1,{ }^{* *}}$ \\ ${ }^{1}$ Renmin Hospital of Wuhan University, Wuhan, Hubei, China \\ "Corresponding author: Renmin Hospital of Wuhan University, Wuhan, Hubei, China. Email: 983673554@qq.com \\ ${ }^{* *}$ Corresponding author: Renmin Hospital of Wuhan University, Wuhan, Hubei, China. Email: 414143244@qq.com \\ Received 2018 August 15; Revised 2019 April 01; Accepted 2019 April 03.
}

\begin{abstract}
Background: Advances in Imaging has been used increasingly to assess coronary lumen and myocardial perfusion with more accuracy.

Objectives: The aim of this study was to evaluate the diagnostic performance of one-stop cardiac CT myocardial perfusion imaging (CT-MPI) combined with coronary artery CT angiography (CCTA) for the detection of functionally relevant myocardial ischemia of aortic valve degeneration (AVD). The study was approved by the patient and passed by the ethics committee.

Methods: This study was a case-control study using random sampling. A total of 109 (109/480) patients suspected of CAD were prospectively enrolled from January 2017 to December 2018 with chest pain as their chief complaint in Renmin Hospital of Wuhan University, Wuhan, Hubei, China. All patients underwent CT-MPI and CCTA on a wide detector Revolution CT. Myocardial blood flow (MBF) was quantified and compared between normal perfusion and abnormal myocardial segments based on AHA-17-segment model. CCTA images were evaluated qualitatively based on SCCT-18-segment model. Radionuclide myocardial perfusion imaging (MPI) is the gold standard; the diagnostic accuracy of CT-MPI, CCTA, and CT-MPI combination with CCTA in the diagnosis of myocardial ischemia of AVD with hemodynamic stenosis was evaluated.

Results: All patients underwent CCTA combined with CT-MPI examination, 69 patients $(63.30 \%)$ with 182 myocardial segments(15. $52 \%$ ) were detected with decreased blood perfusion, the MBF value was lower than that of normal segments [(83.06 \pm 20.11$) \mathrm{mL} .100$ $\mathrm{mL}^{-1} \cdot \mathrm{min}^{-1}$ vs. $\left.(120.02 \pm 28.71) \mathrm{mL} .100 \mathrm{~mL}^{-1} \cdot \mathrm{min}^{-1} ; \mathrm{t}=14.361, \mathrm{P}<0.001\right]$. There were 1914 segments $(97.56 \%)$ that could be used for the diagnosis in CCTA, 302 (15.78\%) segments have clinical significance in this study. Compared with the MPI, the diagnostic sensitivity, specificity, positive and negative predictive value of CT-MPI for myocardial ischemia of AVD were 73.33\%, 73.68\%, 89.80\%, 70.00\%, respectively, $90.00 \%, 68.42 \%, 88.24 \%, 72.22 \%$ for CCTA, and 88.46\%, 70.59\%, 90.20\%, 66.67\% combined with CT-MPI and CCTA. Logistic regression analysis was performed that the AVD was associated with the detection rate of calcified plaques $(r=5.207, P<0.05)$, and calcification of the aortic valve were associated with the detection of plaques in the three and six segments of coronary arteries $(\mathrm{P}<$ 0.05).

Conclusions: Both good CCTA and CT-MPI images can be obtained at the one-stop scanning by Revolution CT. This can be used for the diagnosis of AVD combined with myocardial ischemia. Revolution CT can accurately measure myocardial hemodynamic changes, and provide imaging evidence for the diagnosis and treatment of aortic valve degeneration.
\end{abstract}

Keywords: Aortic Valve, Coronary, Angiography, Computed Tomography, Coronary Stenosis, Degeneration, Ischemia, Myocardial Perfusion Imaging, Revolution CT

\section{Background}

Aortic valve degeneration (AVD) has become a common type of heart disease in the middle-aged and elderly (1), and it has become one of the major types of cardiac valve disease (2). The disease mainly manifests as aortic valve calcification, which is not simply a degenerative change but rather closely related to adverse cardiovascu- lar events such as coronary heart disease (CHD) or myocardial microcirculation levels. Because of its important influence on the prognosis of acute myocardial infarction, infusion has received more and more attention. Gaemperli's (3) and other studies found that only $50 \%$ of patients with abnormal myocardial perfusion have significant coronary stenosis, and normal perfusion cannot rule out coronary 
heart disease, so combinations of microcirculatory conditions (coronary arterial conditions) and myocardial perfusion are essential to the treatment of patients with ischemic heart disease. It is very important to find a reliable inspection method to assess coronary lumen and myocardial perfusion accurately.

\section{Objectives}

In this study, Revolution CT was used to explore the use of one-stop CT heart imaging in myocardial ischemia of AVD, and to investigate its relevance and disease mechanism.

\section{Methods}

This study was a case-control study. Subjects were recruited from January 2017 to December 2018 with chest pain as their chief complaint in Renmin Hospital of Wuhan University, Wuhan, Hubei, China. The study was approved by the patient and passed by the ethics committee. It was estimated that 150 people would be included in this study. Based on the presence or absence of aortic calcification, the patients were considered as AVD patients $(n=109$, male/female: 58/51; mean age: $68.0 \pm 10.5$ years old). One hundred and nine (109/480) patients who were not suspected of having undergone other examinations and treatment for AVD underwent Revolution CT non-invasive coronary CT angiography (CCTA) and myocardial perfusion (CT myocardial perfusion imaging, CT-MPI). Radionuclide myocardial perfusion imaging (MPI) is the gold standard. Exclusion criteria were as follows: severe cardiovascular malformation, disease, postoperative cardiovascular surgery or intention to undergo such surgery; severe heart, liver, renal insufficiency (including acute myocardial infarction and severe arrhythmia); iodine contrast agent sensitivity; inability of iodine contrast agent to enhance the objects; incomplete clinical data and poor image quality. Three hundred seventy-one patients were excluded.

\subsection{Equipment and Methods}

\subsubsection{Pre-Exam Preparation}

Before the scan, the patient's heart rate and blood pressure were measured, and all patients were given training helium. For heart rate $>90$ beats/min, oral betaxolol was given to reduce the heart rate to less than 70 beats/min.

\subsubsection{Image Acquisition}

Image acquisition used the GE Revolution CT (Xtream Edition, 256 Rows) scan range from $1 \mathrm{~cm}$ below the tracheal carina to the diaphragm plane, the equipment was calibrated by engineer. Tube current automatic $\mathrm{MA}$ modulation technology 400 - $700 \mathrm{mAs} / \mathrm{rot}$, tube voltage 100 - 120 $\mathrm{Kv}$, collimator $140-160 \mathrm{~mm}$, rotation time 0.28 seconds, matrix $1024 \times 1024$, FOV180 $-250 \mathrm{~mm}$, using front gated scanning. CCTA data were collected with the same parameters. A $20 \mathrm{G}$ trocar indwelling in the middle elbow vein of a dual-channel high-pressure syringe was used to infuse 45 to $60 \mathrm{~mL}$ of iohexol at a rate of 4.5 to $5.0 \mathrm{~mL} / \mathrm{s}$, followed by $30 \mathrm{~mL}$ of normal saline at the same rate; the front door was used. The automatic control mode triggered the descending aorta at the level of the pulmonary artery. The key points of deviation were avoided as much as possible. The threshold was $120 \mathrm{Hu}$. The CT-MPI scan procedure was started after the CCTA data acquisition. The trigger point of ECG gating was set at the end of systole (this is the best systolic period, about $250 \mathrm{~ms}$ after the $\mathrm{R}$ peak). Total radiation dose: 1.0 to $1.5 \mathrm{mSv}$.

\subsubsection{Image Reconstruction and Evaluation}

CCTA image reconstruction and evaluation: The original image of CCTA was imported into the GE4.6 postprocessing workstation. Cardiac software was used to select the best phase of the coronary arteries (reconstructive systolic and diastolic phases) for 3D reconstruction including VR, CPR, MPR, and MIP (layers: thickness $2.5 \mathrm{~mm}$, layer spacing $2.5 \mathrm{~mm}$ ).

\subsubsection{Image Evaluation}

The American Society of Cardiology revised coronary segmentation method was used here (4). The coronary arteries were divided into 18 segments, according to the literature, the lumen stenosis degree, $0 \%-25 \%, 25 \%-49 \%$, $50 \%-69 \%, 70 \%-99 \%$, and $100 \%$ were considered nonstenosis, mild stenosis, mild stenosis, moderate stenosis, severe stenosis, and occlusion, that means that CAD-RADS grades $0,1,2,3,4$, and 5 . According to Chinese Radiology, $\geq$ $50 \%$ stenosis (combined CAD-RADS grades 3,4 , and 5 ) were identified as having clinical significance in this study. According to the density of plaque, the calcified plaque (CP), mixed plaque (MP), non-calcified plaque (NCP) were classified as the density of the corresponding segment. Combining the images of each group, lesions with areas larger than $1 \mathrm{~mm}^{2}$ and CT values $\geq 130$ Hu were defined as calcification lesions, and calcification observed on any aortic valve leaflets was regarded as AVD (Figure 1).

\subsubsection{Reconstruction and Evaluation of CT-MPI Images}

Perfusion images were taken from 10 to 15 images at different points in time in each section of the perfusion scan and transferred to a GE4.6 post-processing workstation. Perfusion analysis was performed by selecting "Perfusion" 

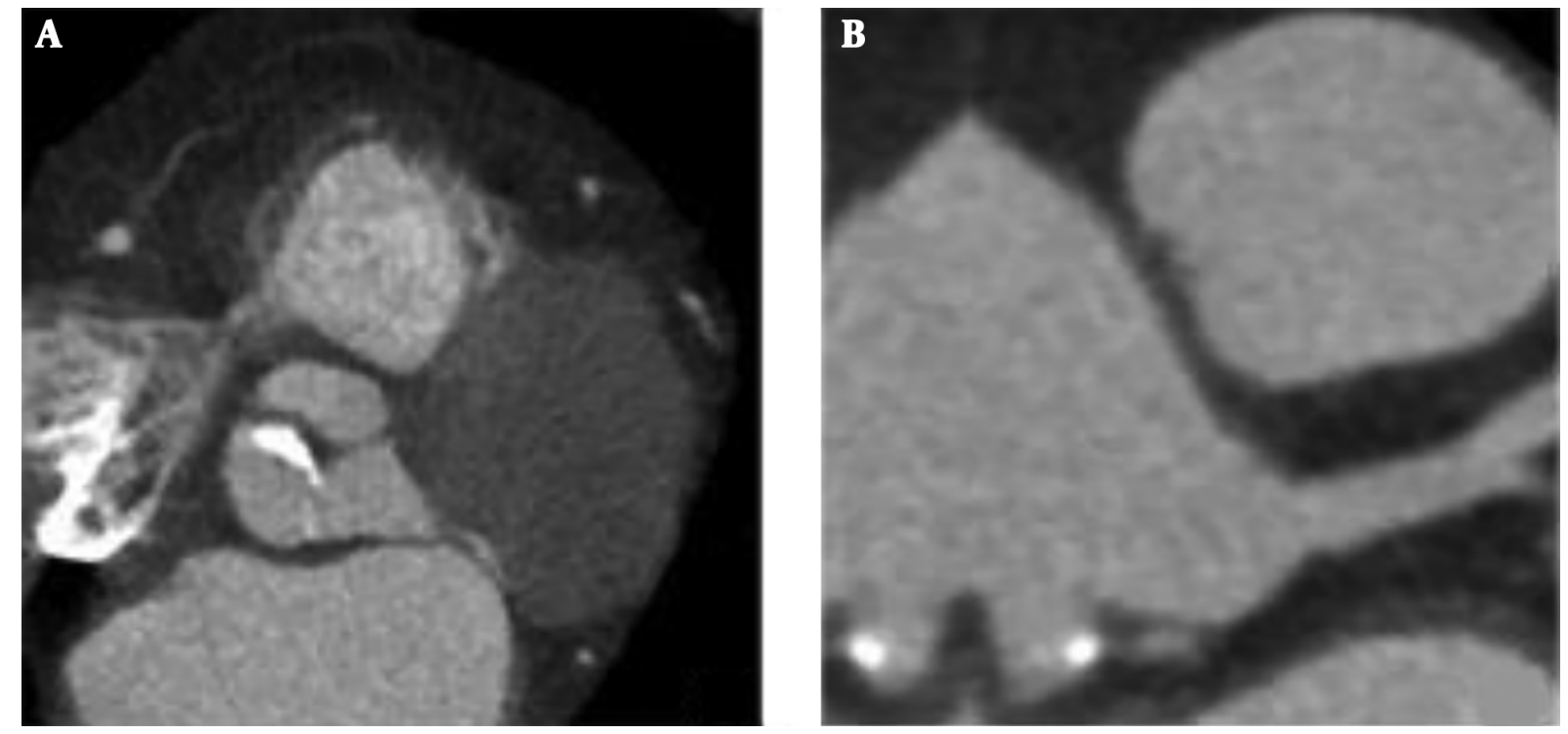

Figure 1. Aortic valve calcification (A, Aortic valve calcification in axial CT imaging; B, Aortic valve calcification in MPR CT imaging)

in the "Body Tumor." Heart three-dimensional color picture. Image evaluation is based on the American Heart Association (AHA) myocardial 17-segment model (5): anterior wall, anterior partition, apical $(1,2,7,8,13,14,17)$ blood supply by LAD, inferior wall. The lower septum $(3,4,9,10,15)$ is supplied by RCA, and the LCX dominates the inferior and anterior sidewalls $(5,6,11,12,16)$. The results were evaluated on the long axis and short axis at the same time. Through the visual evaluation method, the enhanced density of the myocardium in the systolic area of interest was found to be significantly lower than that of the surrounding normal myocardium, whereas the density of the myocardium enhanced in the diastolic area of interest and the surrounding normal myocardium. No significant difference in density was considered an ischemic area. At the same time, the myocardial blood flow (MBF) of each segment was measured against a heart color chart. The measurement of the results of the perfusion area was performed with the exception of the endocardium of the adjacent left ventricle and the area $1 \mathrm{~mm}$ below the pericardium, left ventricular wall thickness $<5 \mathrm{~mm}$ is also considered as ischemic area (excluding apical). According to Chinese Radiology, the definition of stenosis greater than or equal to $50 \%$ is considered coronary stenosis with hemodynamic changes.

MPI images were obtained using the GE Infinia VC Hawkeye 4 SPECT/CT device. The gated acquisition technique was used to capture the image with the R-wave of the electrocardiogram as the trigger potential. The probe was rotated 180 degrees and rotated from the right anterior oblique position. To the left rear oblique position, ev- ery 6 degrees, 1 frame is acquired, and $25 \mathrm{~S}$ is acquired per frame. The raw data was processed using ECTool-box software from GE's Xeleris 3 workstation. According to the unified standard operation, the left ventricular region of interest is semi-automatically delineated and adjusted on the projection map of the original data, and the region of interest is required to include only the entire left ventricle, and the anchor point is located at the center of the left ventricle short axis (determined by the radius value) The apex of the vertical long axis and the horizontal long axis are solid circles, and the base portion is "anti-C" shape. Then, the left ventricular short axis, the horizontal long axis and the vertical long axis myocardial perfusion tomography were obtained by computer automatic processing, showing the location and extent of myocardial perfusion reduction, and the 17-segment bullseye was obtained by projection of the left ventricular short axis.

These images were evaluated by the visual method by two experienced senior diagnostic radiologists with more than 5 years of work experience. After the disputed judgments, both parties reached a final conclusion after discussion. After consistency test, Kendall's Tb coefficient $=0.815$ $(\mathrm{P}<0.001)$, which was closed to the weighted Kappa coefficient $(0.803, \mathrm{P}<0.001)$, suggested that two radiologists had high consistency in disease diagnosed in 109 subjects.

\subsection{Statistical Analysis}

Using IBM SPSS Statistics for Windows, version 22 (IBM Corp., Armonk, N.Y., USA), the clinical data firstly tested for normal distribution. If the normal distribution is met, the 
measurement data is expressed as $\mathrm{x} \pm \mathrm{s}$ or median (M), and the count data is expressed as (n/\%), using the $\chi^{2}$ test. All data in this study is in a normal distribution. The general situations of all subjects were compared by $t$-test, including gender and age; there were count data and appeared as normal assumptions. Perfusion values of perfused normal myocardial segments and perfused abnormal myocardial segments in CT-MPI were compared using independent sample $t$-test. The perfusion abnormalities observed by MPI were used as reference standards to calculate the sensitivity, specificity, positive predictive value, negative predictive value and accuracy of CT-MPI, CCTA, and CT-MPI combined with CCTA for myocardial ischemia diagnosis of each segment. The aortic valve performance and the occurrence of $\mathrm{CP}, \mathrm{NCP}$, and MCP and the segmental and severe stenosis of coronary artery plaque were assessed using logistic regression analysis. These results were statistically significant at $\mathrm{P}<0.05$.

\section{Results}

\subsection{CT-MPI Results}

At CT-MPI images, among 109 patients underwent CCTA combined with CT-MPI examination, 69 patients (63.30\%) with 182 myocardial segments (15.52\%) were detected with decreased perfusion, the MBF value was lower than that of normal segments $\left[(83.06 \pm 20.11) \mathrm{Ml} .100 \mathrm{~mL}^{-1} \cdot \mathrm{min}^{-1} \mathrm{vs}\right.$. $(120.02 \pm 28.71) \mathrm{mL} .100 \mathrm{~mL}^{-1} . \mathrm{min}^{-1} ; \mathrm{t}=14.361, \mathrm{P}<0.001$ ] (Figure 2), as shown in Tables 1 and 2 for comparison with the MPI results.

\subsection{CCTA Results}

A total of 1914 coronary segments can be used for diagnosis in CCTA. According to the diagnostic criteria, CADRADS grades $0,1,2,3,4$, and 5 have 847 (44.2\%), 500 (26.1\%), 265 (13. 8\%), 98 (5.1\%), 110 (5.7\%), and 94 (4.9\%), respectively, $302(15.78 \%)$ segments have clinical significance in this study, as shown in Tables 1 and 2 for comparison with the MPI results.

\subsection{One-Stop Diagnostic Accuracy Evaluation Based on MPI}

Among the 109 patients, MPI showed 69 patients with perfusion defect, case-based CT-MPI, CCTA alone, and combined CT-MPI and CCTA comparison of MPI results are shown in Tables 1 and 2 and Figure 2. Results CCTA stenosis and CT-MPI perfusion defects that were inconsistent with MPI were considered false positives. Comparison of different inspection methods of CAD performance is shown in Tables 1 and 2 .
4.4. Analysis of the Relationship Between Aortic Valve Calcification and Occurrence of Coronary Plaque and Its Luminal Stenosis in AVD Patients

Logistic regression analysis was performed the calcification of the aortic valve was associated with the detection of plaques in the 3 and 6 segments of coronary arteries $(\mathrm{P}<0.05)$ (Table 3 and Figure 3 ). Logistic regression analysis was also performed on coronary plaque and aortic valve calcification in different segments. The results showed that the AVD was associated with the detection rate of calcified plaques $(r=5.207, \mathrm{P}<0.05)$. There was no correlation between the AVD and other observational indicators $(P>0.05)$ (Table 4).

\section{Discussion}

AVD has become one of the major types of CAD, and $\mathrm{CAD}$ is a form of ischemic myocardial damage caused by an imbalance between coronary blood flow and myocardial oxygen demand due to coronary atherosclerosis or functional changes (6). Patients with coronary atherosclerosis may present with multiple myocardial pathological changes, including myocardial ischemic injury including reversibility and irreversibility; the irreversible myocardial injury is myocardial infarction; reversible injury refers to contraction due to myocardial ischemia (7). The myocardium is reduced but remains viable. The structural integrity of surviving myocardial cell membranes, mitochondria, and other structures contributes to the continuation of glucose metabolism and can provide myocardial contractile reserve (8). After myocardial infarction is removed and the area undergoes vascular reconstruction, all myocardial functions that have not been completely destroyed will improve. Therefore, prospective assessment of viable myocardium is of great significance for the choice of treatment. Early detection of ischemic myocardium is important since It can improve the prognosis of ischemic heart disease (9). At present, the diagnosis of CAD is mostly made through CCTA to assess the stenosis of blood vessels, but CCTA often overestimates the degree of coronary artery stenosis, which means some patients receive unnecessary invasive ICA examination. Myocardial perfusion is assessed primarily by radionuclide or MRI myocardial perfusion; however, there is a non-linear correlation between the degree of coronary artery stenosis and the degree of myocardial ischemia (10). These examination methods have certain limitations, and they cannot simultaneously produce images of coronary and myocardial perfusion, that means they can do it but not simultaneously. For this reason, it is essential to find a way to accurately obtain coronary artery lumen images and myocardial perfusion images in a onestop way. 


\begin{tabular}{lccccccc}
\hline Table 1. One-Stop Diagnostic Results Based on MPI (People) & \multicolumn{3}{c}{} & & & \\
\hline MPI & \multicolumn{2}{c}{ CT-MPI } & & CCTA & \multicolumn{2}{c}{ CT-MPI + CCTA } \\
\cline { 2 - 8 } & Positive & Negative & Positive & Negative & Positive & Negative \\
\hline Positive & 44 & 6 & 45 & 5 & 46 & 6 \\
Negative & 5 & 14 & 6 & 13 & 5 & 12 \\
Total & 49 & 20 & 51 & 18 & 51 & 18 \\
\hline
\end{tabular}

Abbreviations: CCTA, CT angiography; CT-MPI, CT myocardial perfusion imaging; MPI, myocardial perfusion imaging.

\begin{tabular}{lcccc}
\hline \multicolumn{1}{l}{ Table 2. One-Stop Diagnostic Accuracy Evaluation Based on MPI (\%) } & & & \\
\hline Methods & Sensitivity & Specificity & PPV & NPV \\
\hline CT-MPI & 73.33 & 73.68 & 89.80 & 70.00 \\
CCTA & 90.00 & 68.42 & 88.24 & 72.22 \\
CT-MPI +CCTA & 88.46 & 70.59 & 90.20 & 66.67 \\
\hline
\end{tabular}

Abbreviations: CCTA, CT angiography; CT-MPI, CT myocardial perfusion imaging; MPI, myocardial perfusion imaging.

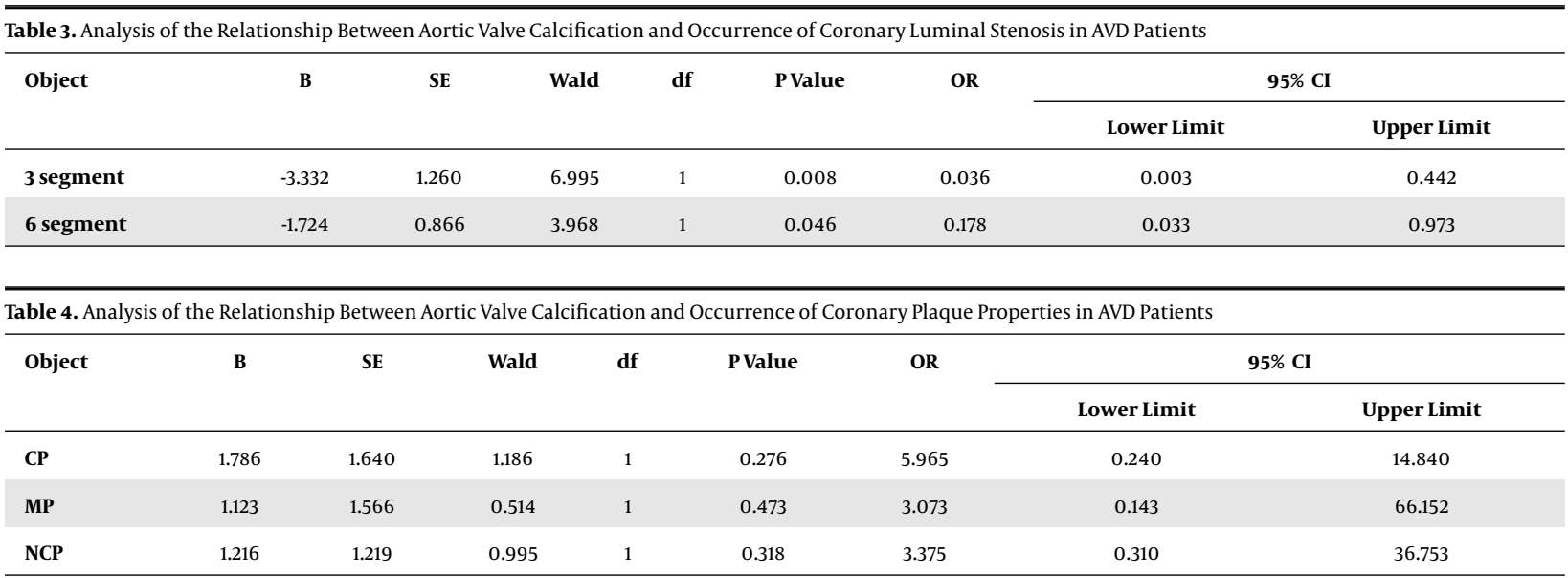

Abbreviations: CP, calcified plaque; MP, mixed plaque; NCP, non-calcified plaque.

Calcification of heart valves is more likely to occur in the aortic valve area (11). This is mainly due to the lower shear stress in the aortic sinus stenosis, which can develop into $\mathrm{CP}$ according to the fibrous plaque degeneration and calcium deposition of the valve tissue (12). For these reasons, clinical CP is considered a characteristic of AVD, but CP may also appear in CHD (13). AVD and CHD have many similarities in risk factors and pathological changes, but they are not entirely the same (14). The research performed an average of 5 years of follow-up for 8401 patients without obvious CHD and AVD and found that the overall mortality rate of AVD patients was significantly higher than that of patients without AVD, suggesting that AVD may be considered an independent predictor of adverse cardiovascular events; AVD can also be regarded as a predictor of CHD, Jassal et al. reported similar findings (15). This article may facilitate early warning of the occurrence of CHD and pro- vide a foundation for imaging suitable for early clinically accurate diagnosis and early treatment.

This article mainly uses Revolution CT, performing comprehensive morphological observation and assessment on the aortic valve and coronary artery. The CT equipment has the following advantages: It uses post-processing software for multi-directional observation. Calcium integration measurement can also effectively avoid ultrasound measurement errors caused by calcification, so as to better serve clinical treatment. Since the equipment used in this study has a wider body and can perform volume axis scanning and flexible spiral scanning more quickly which is not possible by traditional CT; the equipment in this study only requires one injection of contrast agent and one heartbeat. Within a single time period, anatomical heart information, plaque composition information, myocardial perfusion, and cardiac function can be obtained, and a wide range 

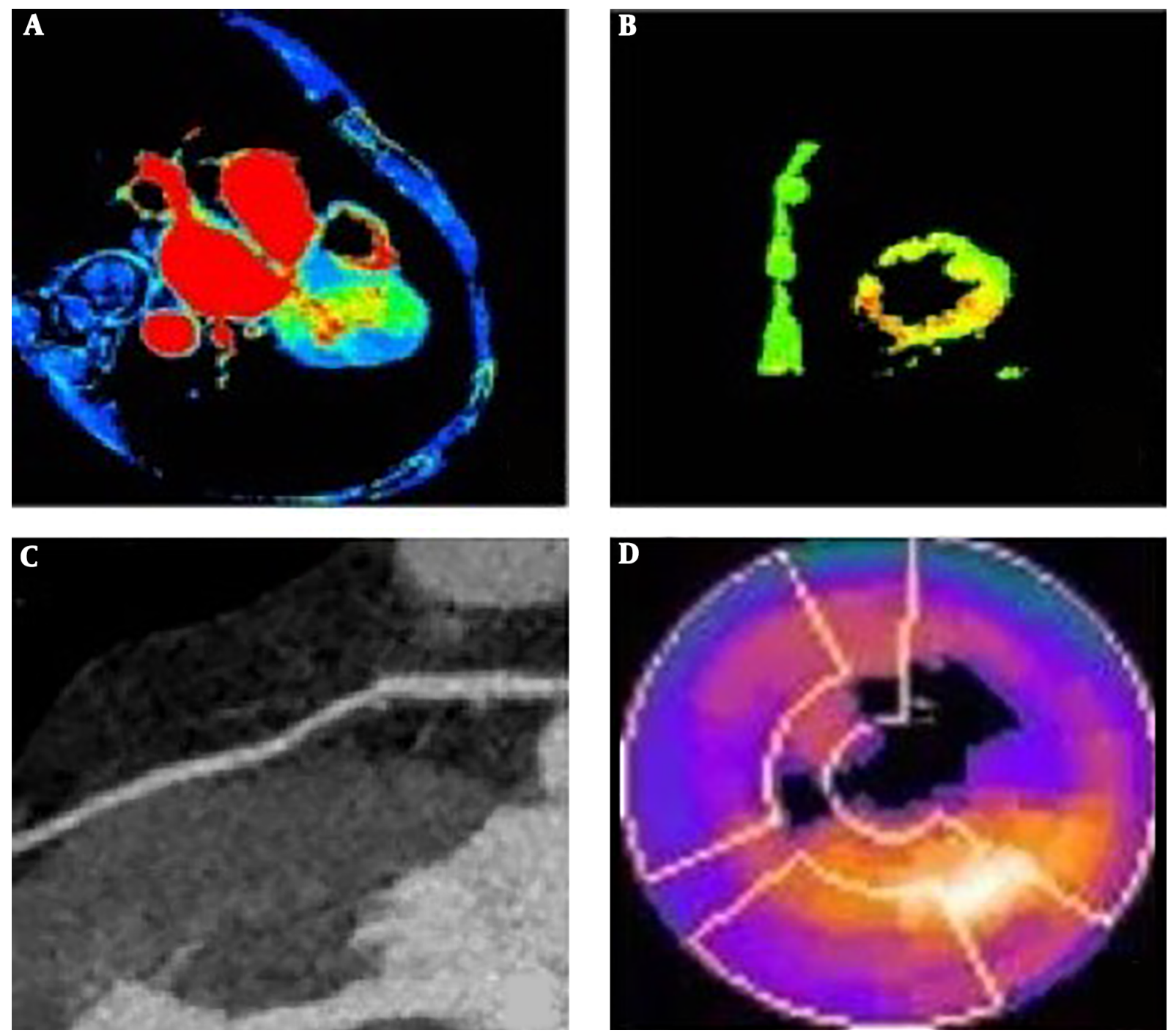

Figure 2. Revolution CT one-stop cardiac CT (CT-MPI combined with CCTA) can detect myocardial perfusion defects and hemodynamic changes in AVD. (A and B, The MBF values of hypo-perfused myocardial segments decreased in the AVD group; C, Stenosis of the segment 6 of the coronary artery and corresponding segmental myocardial perfusion reduction; d. MPI shows mild myocardial ischemia in the left ventricular apex and septal apical segment).

of vascular images can be produced with a small dose, so the patient is not exposed to any unnecessary radiation. Although this method required more medical resources, patient compliance with the data collection of the study was improved, image quality was ensured, improving the reliability of the research data. Using one shot at a time to enhance one scan does not increase medical expenses or medical resources.

In this study, the AVD was associated with the detection rate of calcified plaques. This may be because AVD combined with CP has a relationship with anatomy and hemodynamics (16). This was consistent with the results reported by Zhang et al. (5). Some studies have also shown that most patients with AVD and CP have sudden valve sinus lesions (17). In addition, the pathogenesis of degenerative changes in the elderly is the diastolic pressure of the aortic sinus systolic decreasing at the aortic valve. Excessive load (18), which stimulates the thickening of the valve calcification site, causing valve dysfunction, ultimately affects the opening and closing of the normal valve, and then increases the left ventricular work and myocardial oxygen consumption, easily leading to the occurrence of CHD, having a poor prognosis $(19,20)$. Some scholars believe that aortic insufficiency may be related to changes in 

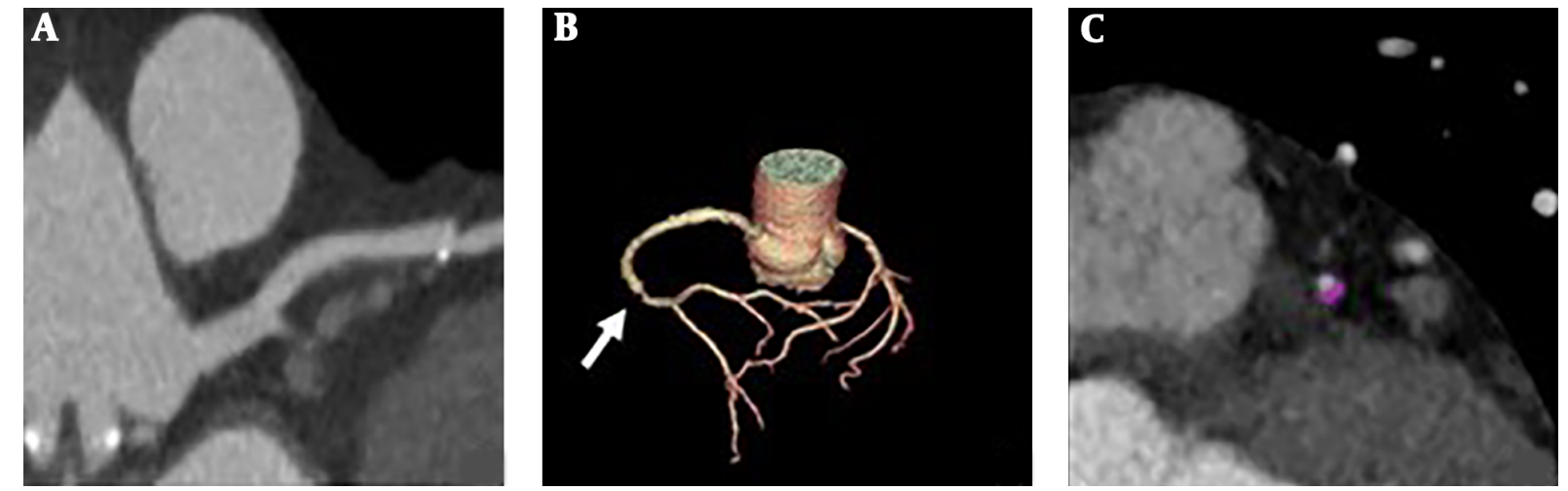

Figure 3. Calcification of aortic valve and detection of a calcified plaque in coronary artery in AVD. (A, Calcification of the aortic valve in the AVD group is associated with the detection of coronary segment 6 ; B, The segment 3 of the coronary arteries are related to the stenosis of the lumen of the corresponding segment; $C$, Aortic valve calcification and coronary artery calcification in the AVD).

left heart function and coronary heart disease. In clinical work, some patients with aortic valve degenerative disease have active valve insufficiency (21). However, in this study, no obvious morphological abnormalities other than calcification of the valve appeared, which may be related to the milder symptoms of the subjects included in this study, and there was no significant change in cardiac function. This will continue to expand in subsequent studies.

In this study, there was no statistically significant difference in gender among patients, however, the correlation between aortic valve calcification and coronary arteriosclerosis detection indicates that coronary arteriosclerosis is not only a degenerative change but also involves inflammation. The expression of factors such as adipose factor and other factors has confirmed that the aortic valve is thickened (22). In addition to fibrous tissue and nodular calcification, there are macrophages and other inflammatory cells, and nearby fatty tissue (23). The classical mechanisms of regional inflammatory response and abnormal lipid metabolism related to the occurrence and development of AVD (24). Abnormal lipid metabolism can induce or aggravate the inflammatory response of the valve and promote AVD progression (25). An increase in the incidence of diseases such as metabolic syndrome may also lead to progression of the disease. The regulation of noncoding RNA can also affect the expression of related factors (26). Molecular biology studies indicate that $\operatorname{Lp}(\mathrm{a})$ encoded gene SNPs cause abnormal lipid metabolism, and miRNA and IncRNAs regulate calcification-related genes and AVD (27). With regard to progress, miRNA and lnc RNA up-regulate inflammatory factors that promote calcification while inhibiting the activity of anti-calcification factors and so ultimately accelerate the progression of valve calcification (28). The results of this study are consistent with these reports. This merits attention during clinical diagnosis and treatment. AVD should not be treated as a degenerative change. Risk assessment and early intervention in asymptomatic AVD patients can prevent serious cardiovascular disease.

In this study, some AVD patients showed abnormal myocardial perfusion, but there was no obvious abnormality in myocardial perfusion in others. Obvious morphological changes were observed in some AVD patients also, which verified the morphological changes reported in previous studies. There was a non-linear correlation with myocardial perfusion (20). Myocardial perfusion refers to the volume of blood flow per unit volume of the myocardium per unit time (29). The flow of blood from the small arteries into the blood vessels through the capillaries, which can express the function and metabolism of the cardiomyocytes (30). The change in resting myocardial perfusion volume showed a certain correlation with the degree of coronary artery stenosis (31). When an iodine contrast agent was used to enhance the examination, normal myocardial tissue, ischemic myocardial tissue, and myocardial tissue with myocardial infarction had different contrast agent uptake capabilities (32). Healthy myocardial tissue is normal and the amount of ischemic myocardium first increases and then decreases. The infarcted myocardium was not enhanced, and the different distribution of the contrast agent in the myocardial tissue was expressed with a pseudo-colored image, producing an image of myocardial perfusion. Because the load is limited, some patients cannot tolerate exercise, and the system is inconvenient to operate, we here used a bolus of iodine contrast agent instead. We performed a rapid dynamic scan and acquire a time-density curve to quantitatively assess tissue perfusion (33). CT-MPI was initially performed using elec- 
tron beam CT and multi-slice spiral CT, but the electron beam CT was limited by the spatial resolution, which led to the details of the image being displayed unclear; with the development of CT technology, especially in recent years The high-end CTs that have emerged one after another have achieved preliminary results in the clinical study of CT myocardial perfusion (34).

In this study, combined CT-MPI and CCTA improved the positive predictive value of myocardial perfusion abnormalities, indicating that the two have complementary effects, similar to the results reported (35), and performed a 320-MDCT multicenter trial, with blood, detection of vascular stenosis with abnormal dynamics, and one-stop combined diagnosis (36). To a large extent, they found and ruled out CCTA's false positive results, and in patients with suspected myocardial ischemia, reduced unnecessary invasive tests. The functional information obtained by CT-MPI is a good complement to CCTA inspections. This also prompted us to develop a CAD one-stop CT diagnosis model.

\subsection{Conclusions}

In this study, we found a way to accurately obtain coronary artery lumen images and myocardial perfusion images in a one-stop way. This means that we can obtain information about anatomy and function of AVD at one exam. Only a single contrast injection can be used to obtain good coronary and myocardial perfusion imaging. The morphological and functional information of the heart is provided completely without increasing the amount of contrast agent and the lower total radiation dose and shorter time. It also has high accuracy in the diagnosis of coronary heart disease, and it is expected to become a "onestop" diagnosis method for coronary heart disease, providing new ideas for the choice of clinical treatment methods and therapeutic observation.

Limitations of this study: (1) Subjects in this study have relatively mild symptoms and fewer interventions. Future studies should be performed on patients with more severe symptoms. (2) This is a single-center study with a relatively small sample size. In the future, it is necessary to improve and conduct in-depth research on the multi-center work of large sample groups.

Both good CCTA and CT-MPI images can be obtained at the one stop scanning by Revolution CT, which can be used for the diagnosis of AVD combined with myocardial ischemia. Revolution CT can accurately measure myocardial hemodynamic changes, and provide imaging evidence for the diagnosis and treatment of aortic valve degeneration. Application of the One-stop Cardiac CT scan (myocardial perfusion imaging combined with CCTA) on wide detector Revolution CT can provides anatomical and hemodynamic information in one examination, providing an imaging basis for timely detection, treatment, and prevention of CAD.

\section{Footnotes}

Conflict of Interests: It is not declared by the authors. Funding/Support: It is not declared by the authors. Patient Consent: It is not declared by the authors.

\section{References}

1. Driessen RS, Stuijfzand WJ, Raijmakers PG, Danad I, Min JK, Leipsic JA, et al. Effect of plaque burden and morphology on myocardial blood flow and fractional flow reserve. J Am Coll Cardiol. 2018;71(5):499-509. doi: 10.1016/j.jacc.2017.11.054. [PubMed: 29406855].

2. Ahmadi A, Leipsic J, Ovrehus KA, Gaur S, Bagiella E, Ko B, et al. Lesionspecific and vessel-related determinants of fractional flow reserve beyond coronary artery stenosis.JACC Cardiovasc Imaging. 2018;11(4):52130. doi:10.1016/j.jcmg.2017.11.020. [PubMed: 29311033].

3. Nerlekar N, Ha FJ, Cheshire C, Rashid H, Cameron JD, Wong DT, et al. Computed tomographic coronary angiography-derived plaque characteristics predict major adverse cardiovascular events: A systematic review and meta-analysis. Circ Cardiovasc Imaging. 2018;11(1). e006973. doi: 10.1161/CIRCIMAGING.117.006973. [PubMed: 29305348].

4. Doherty JU, Kort S, Mehran R. 2017 appropriate use criteria for multimodality imaging in valvular heart disease. Cardiovasc Comput Tomogr. 2017;10(24):122-36.

5. Zhang YD, Li M, Qi L, Wu CJ, Wang X. Hypertrophic cardiomyopathy: Cardiac structural and microvascular abnormalities as evaluated with multi-parametric MRI. Eur J Radiol. 2015;84(8):1480-6. doi: 10.1016/j.ejrad.2015.04.028. [PubMed: 26003194].

6. Ziadi MC. Myocardial flow reserve (MFR) with positron emission tomography (PET)/computed tomography (CT): Clinical impact in diagnosis and prognosis. Cardiovasc Diagn Ther. 2017;7(2):206-18. doi: 10.21037/cdt.2017.04.10. [PubMed: 28540215]. [PubMed Central: PMC5422840].

7. Pontone G, Rabbat MG, Guaricci AI. Stress computed tomographic perfusion: Are we ready for the green light? Circ Cardiovasc Imaging. 2017;10(4). doi: 10.1161/CIRCIMAGING.117.006324. [PubMed: 28389508].

8. Yang DH, Kim YH, Roh JH, Kang JW, Ahn JM, Kweon J, et al. Diagnostic performance of on-site CT-derived fractional flow reserve versus CT perfusion. Eur Heart J Cardiovasc Imaging. 2017;18(4):432-40. doi: 10.1093/ehjci/jew094. [PubMed: 27354345].

9. Rajamannan NM. Calcific aortic valve disease in familial hypercholesterolemia: The LDL-density-gene effect. J Am Coll Cardiol. 2015;66(24):2696-8. doi: 10.1016/j.jacc.2015.10.011. [PubMed: 26700831].

10. Coenen A, Rossi A, Lubbers MM, Kurata A, Kono AK, Chelu RG, et al. Integrating CT myocardial perfusion and CT-FFR in the work-up of coronary artery disease. JACC Cardiovasc Imaging. 2017;10(7):760-70. doi: 10.1016/j.jcmg.2016.09.028. [PubMed: 28109933].

11. Pontone G, Andreini D, Guaricci AI, Guglielmo M, Mushtaq S, Baggiano A, et al. Rationale and design of the perfection (comparison between stress cardiac computed tomography perfusion versus fractional flow reserve measured by computed tomography angiography in the evaluation of suspected coronary artery disease) prospective study. J Cardiovasc Comput Tomogr. 2016;10(4):330-4. doi: 10.1016/j.jcct.2016.03.004. [PubMed: 27050025].

12. Wei F, Sun N, Cai C, Feng S, Tian J, Shi W, et al. Associations between serum uric acid and the incidence of hypertension: A Chinese senior 
dynamic cohort study. J Transl Med. 2016;14(1):110. doi: 10.1186/s12967016-0866-0. [PubMed: 27129957]. [PubMed Central: PMC4851787].

13. Matveeva A, Schmitt RR, Edtinger K, Wagner M, Kerber S, Deneke T, et al. Coronary $\mathrm{CT}$ angiography in patients with atrial fibrillation: Standard-dose and low-dose imaging with a high-resolution wholeheart CT scanner. Eur Radiol. 2018;28(8):3432-40. doi: 10.1007/s00330017-5282-1. [PubMed: 29426987].

14. Blaha MJ, Budoff MJ, Rivera JJ, Khan AN, Santos RD, Shaw LJ, et al. Relation of aortic valve calcium detected by cardiac computed tomography to all-cause mortality. Am J Cardiol. 2010;106(12):1787-91. doi: 10.1016/j.amjcard.2010.08.019. [PubMed: 21055710].

15. Ten Kate GR, Bos S, Dedic A, Neefjes LA, Kurata A, Langendonk JG, et al. Increased aortic valve calcification in familial hypercholesterolemia: Prevalence, extent, and associated risk factors. J Am Coll Cardiol. 2015;66(24):2687-95. doi: 10.1016/j.jacc.2015.09.087. [PubMed: 26700830].

16. Andreini D, Pontone G, Mushtaq S, Mancini ME, Conte E, Guglielmo M, et al. Image quality and radiation dose of coronary $\mathrm{CT}$ angiography performed with whole-heart coverage CT scanner with intra-cycle motion correction algorithm in patients with atrial fibrillation. Eur Radiol. 2018;28(4):1383-92. doi: 10.1007/s00330-017-5131-2. [PubMed: 29164383].

17. Kang SJ, Yang DH, Koo HJ, Yun SC, Lee JG, Kang JW, et al. Intravascular ultrasound-derived morphological predictors of myocardial ischemia assessed by stress myocardial perfusion computed tomography. Catheter Cardiovasc Interv. 2017;89(7):E207-16. doi: 10.1002/ccd.26710. [PubMed: 27567025].

18. Bosse K, Hans CP, Zhao N, Koenig SN, Huang N, Guggilam A, et al. Endothelial nitric oxide signaling regulates Notch1 in aortic valve disease. J Mol Cell Cardiol. 2013;60:27-35. doi: 10.1016/j.yjmcc.2013.04.001. [PubMed: 23583836]. [PubMed Central: PMC4058883].

19. Myles V, Liao J, Warnock JN. Cyclic pressure and angiotensin II influence the biomechanical properties of aortic valves.J Biomech Eng. 2014;136(1):11011. doi: 10.1115/1.4026041. [PubMed: 24240552].

20. Keir M, Spears D, Caldarone C, Crean AM. Proving the innocence of a "malignant" coronary artery: Calling dobutamine stress CT for the defence!. J Cardiovasc Comput Tomogr. 2017;11(1):68-9. doi: 10.1016/j.jcct.2016.07.006. [PubMed: 27431606].

21. Latif N, Sarathchandra P, Chester AH, Yacoub MH. Expression of smooth muscle cell markers and co-activators in calcified aortic valves. Eur Heart J. 2015;36(21):1335-45. doi: 10.1093/eurheartj/eht547. [PubMed: 24419809].

22. Venardos N, Nadlonek NA, Zhan Q, Weyant MJ, Reece TB, Meng X, et al. Aortic valve calcification is mediated by a differential response of aortic valve interstitial cells to inflammation. J Surg Res. 2014;190(1):1-8. doi: 10.1016/j.jss.2014.03.051. [PubMed: 24746950]. [PubMed Central: PMC4053510].

23. Albrecht MH, Nance JW, Schoepf UJ, Jacobs BE, Bayer R2, Litwin SE, et al. Diagnostic accuracy of low and high tube voltage coronary CT angiography using an X-ray tube potential-tailored contrast medium injection protocol. Eur Radiol. 2018;28(5):2134-42. doi: 10.1007/s00330017-5150-z. [PubMed: 29181587].

24. Pelgrim GJ, Duguay TM, Stijnen JM, Varga-Szemes A, Van Tuijl $S$, Schoepf UJ, et al. Analysis of myocardial perfusion parameters in an ex-vivo porcine heart model using third generation dual-source CT. J Cardiovasc Comput Tomogr. 2017;11(2):141-7. doi: 10.1016/j.jcct.2017.01.012. [PubMed: 28202246].

25. Li Y, Yu M, Li W, Lu Z, Wei M, Zhang J. Third generation dual- source CT enables accurate diagnosis of coronary restenosis in all size stents with low radiation dose and preserved image quality. Eur Radiol. 2018;28(6):2647-54. doi: 10.1007/s00330-017-5256-3. [PubMed: 29349698].

26. Leipsic J, Abbara S, Achenbach S, Cury R, Earls JP, Mancini GJ, et al. SCCT guidelines for the interpretation and reporting of coronary CT angiography: A report of the Society of Cardiovascular Computed Tomography Guidelines Committee. J Cardiovasc Comput Tomogr. 2014;8(5):342-58. doi: 10.1016/j.jcct.2014.07.003. [PubMed: 25301040].

27. Danad I, Szymonifka J, Schulman-Marcus J, Min JK. Static and dynamic assessment of myocardial perfusion by computed tomography. Eur Heart J Cardiovasc Imaging. 2016;17(8):836-44. doi: 10.1093/ehjci/jew044. [PubMed: 27013250]. [PubMed Central: PMC4955293].

28. Pontone G, Muscogiuri G, Andreini D, Guaricci AI, Guglielmo M, Mushtaq S, et al. The new frontier of cardiac computed tomography angiography: Fractional flow reserve and stress myocardial perfusion. Curr Treat Options Cardiovasc Med. 2016;18(12):74. doi: 10.1007/s11936-016-0493-3. [PubMed: 27783337].

29. Udholm PM, Linde JJ, Barton R, Kuhl JT, Hove JD, Sorgaard M, et al. Functional impact of atherosclerosis on epicardial coronary conductance vessels assessed With MDCT. JACC Cardiovasc Imaging. 2017;10(4):490-1. doi: 10.1016/j.jcmg.2016.03.004. [PubMed: 27209108].

30. Penagaluri A, Higgins AY, Vavere AL, Miller JM, Arbab-Zadeh A, Betoko A, et al. Computed tomographic perfusion improves diagnostic power of coronary computed tomographic angiography in women: Analysis of the CORE320 trial (coronary artery evaluation using 320row multidetector computed tomography angiography and myocardial perfusion) according to gender. Circ Cardiovasc Imaging. 2016;9(11). doi: 10.1161/CIRCIMAGING.116.005189. [PubMed: 27811151].

31. Michelsen MM, Mygind ND, Frestad D, Prescott E. Women with stable angina pectoris and no obstructive coronary artery disease: Closer to a diagnosis. Eur Cardiol. 2017;12(1):14-9. doi: 10.15420/ecr.2016:33:2. [PubMed: 30416544]. [PubMed Central: PMC6206464].

32. Kuhl JT, George RT, Mehra VC, Linde JJ, Chen M, Arai AE, et al. Endocardial-epicardial distribution of myocardial perfusion reserve assessed by multidetector computed tomography in symptomatic patients without significant coronary artery disease: insights from the CORE320 multicentre study. Eur Heart J Cardiovasc Imaging. 2016;17(7):779-87. doi: 10.1093/ehjci/jev206. [PubMed: 26341292]. [PubMed Central: PMC5006103].

33. Ladeiras-Lopes R, Bettencourt N, Ferreira N, Sampaio F, Pires-Morais $\mathrm{G}$, Santos L, et al. CT myocardial perfusion and coronary CT angiography: Influence of coronary calcium on a stress-rest protocol. J Cardiovasc Comput Tomogr. 2016;10(3):215-20. doi: 10.1016/j.jcct.2016.01.013. [PubMed: 26869367].

34. Marini C, Seitun S, Zawaideh C, Bauckneht M, Morelli MC, Ameri $\mathrm{P}$, et al. Comparison of coronary flow reserve estimated by dynamic radionuclide SPECT and multi-detector x-ray CT.J Nucl Cardiol. 2017;24(5):1712-21. doi: 10.1007/s12350-016-0492-5. [PubMed: 27151303].

35. Cademartiri F, Seitun S, Clementeetal A. Myocardialblood ow quanti cation for evaluation of coronary artery disease by computed tomography. Cardiovasc Diagn Ther. 2017;7(2):129-50.

36. Sicari R, Cortigiani L. The clinical use of stress echocardiography in ischemic heart disease. Cardiovasc Ultrasound. 2017;15(1):7. doi: 10.1186/s12947-017-0099-2. [PubMed: 28327159]. [PubMed Central: PMC5361820]. 